\title{
Docencia, investigación y gestión: Reflexiones sobre su papel en la calidad de la educación superior*
}

\author{
Teaching, research and management: Reflections on its role in the quality of higher \\ education
}

\author{
Ensino, pesquisa e gestão: Reflexões sobre o seu papel na qualidade \\ da educação superior*
}

\begin{abstract}
Audin Aloiso Gamboa-Suárez**
Forma de citar: Gamboa, A. A. (2016). Docencia, investigación y gestión: Reflexiones sobre su papel en la calidad de la educación superior. Perspectivas. 1(1). 81-90.
\end{abstract}

Recibido:

Enero 15 de 2016

Aceptado:

Marzo 28 de 2016

\section{Resumen}

El presente artículo de reflexión hace parte de una investigación denominada docencia, investigación y gestión: representaciones de actores educativos sobre las dinámicas de calidad en la UFPS, El escrito hace un análisis de la docencia, la investigación y la gestión como principales determinantes de la calidad de la educación superior. Se muestran algunas de las investigaciones más relevantes en las tres categorías y el papel que cumplen en las dinámicas de calidad en las universidades.

Palabras clave: calidad, docencia, educación superior gestión, investigación.

\begin{abstract}
The present reflection article is part of a research called teaching, research and management: representations of educational actors on the dynamics of quality in the UFPS, The paper makes an analysis of teaching, research and management as the main determinants of the quality of higher education. It shows some of the most relevant research in the three categories and the role they play in the quality dynamics in universities.
\end{abstract}

Keywords: quality, teaching, higher education, management, research.

\section{Resumo}

O presente artigo de reflexão é parte de uma pesquisa chamada ensino, pesquisa e gestão: representações dos atores educativos sobre as dinâmicas de qualidade na UFPS, a carta faz uma análise do ensino, pesquisa e gestão como principais determinantes da qualidade do ensino superior. São apresentadas algunas das pesquisas mais relevantes em todas as três categorias e o papel desempenhado nas dinâmicas de qualidade nas universidades.

Palavras chave: qualidade, ensino, gestão, pesquisa, ensino superior.

\footnotetext{
*Proyecto financiado por el Fondo de Investigaciones de la UFPS según contrato No. 035-2015.

** Docente investigador de la Universidad Francisco de Paula Santander, Doctorando en Ciencias de la Educación (RUDECOLOMBIA), Magíster en Pedagogía (UIS), Director de la Maestría en Práctica Pedagógica de la UFPS e investigador del Grupo en estudios sociales y pedagogía para la paz (GIESPPAZ - Categoría A en Colciencias). Contacto: docaudin@gmail.com
} 
Vol. 1

No. 1

Jul - Dic 2016

ISSN: 2590-9215 (En línea) PP: $81-90$

\section{Docencia universitaria y calidad de la Educación}

La docencia hace parte fundamental de la educación superior y esta infiere directamente en la calidad del servicio que presta la universidad. La calidad de la docencia universitaria "implica un elevado conocimiento sobre cómo funcionan los procesos y las dinámicas de aprendizaje de los sujetos" (Zabalsa \& Zabalsa, 2010, p.58), el docente no es solo una figura o un actor más en las dinámicas universitarias, es uno de los principales protagonistas en la formación de los estudiantes y en la construcción de conocimiento desde la investigación.

Son múltiples las investigaciones que se han realizado sobre la calidad de la docencia en la educación superior, estos estudios dan cuenta entre otros elementos sobre los estilos de enseñanza (Laudadio, 2012; López, 2010 \& De Vincenzi, 2009), modelos de evaluación docente (García et ál, 2010; Manzi, 2011; Tejedor \& García, 2010 y Rueda, 2009) y un tema fundamental, las competencias de los profesores de educación superior (Bozu \& Herrera, 2009; Arrufat, Sánchez, \& Santiuste, 2010; Guzmán \& Marín, 2011 y Tirado, 2009).

En este sentido, son variadas las miradas que se le pueden hacer a un buen profesor: actitudes, formas de evaluar, preparación intelectual, formas de enseñar, actividades de investigación, reflexivos de su propia práctica, prudentes y conjugar la exigencia con la comprensión.

Particularmente, en esta categoría se analizará las competencias de los maestros universitarios, pero primero se abordará de una manera general el concepto de competencias:

\subsection{El concepto de competencias}

Las competencias en el ámbito de la educación superior supone conocer y comprender el saber cómo actuar y al saber cómo ser (Beneitone et al., 2007). Los autores de este informe afirman que las competencias en educación superior es un nuevo enfoque que determina las metas a lograrse en la formación de un profesional $\mathrm{y}$ se fundamentan en un saber profundo, no sólo saber qué y saber cómo, sino saber ser persona en un mundo complejo Cambiante y competitivo. Además de señalar que las competencias en educación son un enfoque novedoso, del mismo modo afirman que es un conjunto de enfoques de aprendizaje, es decir una configuración de diferentes miradas. Hay que analizar entonces si este cumulo de abordajes desde diferentes campos no configuran realmente un modelo estructurado en educación superior. Es importante señalar que en el texto de Beneitone et al., (2007) quienes afirman que las competencias hacen relación a un enfoque, de igual modo señalan se puede considerar como modelo cuando hacen parte de las estructuras curriculares y de unos procesos de formación bien definidos.

Otros autores como Hernandez (2009), señalan que las competencias deben romper con prácticas y formas de pensar propias de sistemas educativos transmisioncitas y tradicionales, esto podría en cierto sentido señalar que las competencias deben permear esos viejos modelos de enseñanza que deben remplazarse no solamente por la inclusión de las competencias si no por el surgimiento de nuevos paradigmas educativos.

Las competencias se refieren a una propuesta que pretende dar solución al problema educativo (Montenegro, 2005) y una interesante reflexión sobre el tema es "el Enfoque Socioformativo Complejo de Tobón, que se diferencia de otras metodologías de diseño curricular por competencias en la medida que integra la investigación acción como eje del cambio educativo y la formación epistemológica en pensamiento complejo" (Tobón \& Tejada, 2006, p. 42). Los mismos autores afirman que el modelo de enseñanza 
basado en competencias profesionales es una metodología que aporta a la práctica pedagógica del Maestro. Tobón (2008) da explicación al enfoque por competencias basado en modelos como el de normalización de unidades, dominios y otros. Sin embargo, el autor da más relevancia al modelo Sistémico Complejo. El cuál se basa en identificar y normalizar las competencias con base en tres componentes: problemas, competencias $\mathrm{y}$ criterios.

Es interesante analizar la obra de Tobón en cuanto al uso de dos términos que se han venido analizando en este escrito: el enfoque y el modeo, la gran mayoría de la obra del autor toma las competencias como un enfoque, sin embargo como se mencinó anteriormente el término modelo aparece esporadicamente en su discurso e incluso de manera combinada cuando señala que "las universidades vienen adoptando este enfoque por competencias después de pasar por los otros modelos, con el fin de hacer más viable la formación y evaluación de competencias desde los maestros y maestras" (Tobón. 2008, p. 7).

En este sentido la evaluació por competencias pretende como lo sugiere Villardón (2006) citando a McDonald y col., (2000)

\begin{abstract}
"asegurar que tanto el proceso de enseñanza-aprendizaje como la evaluación están guiados por los resultados de aprendizaje pretendidos; por otro lado, para facilitar la concesión de créditos por la competencia adquirida en otros contextos; y para ayudar a los estudiantes a comprender lo que se espera de ellos, así como para informar a otras personas interesadas sobre el significado de una calificación determinada" (p.7)
\end{abstract}

Es importante señalar que las competencias en educación superior es un tema relativamente nuevo en Colombia y aunque hay literatura abundante sobre el particular se puede afirmar que aún no se ha configurado como modelo de formación si no por el contario como una mirada utópica de práctica pero bien delimitada teóricamente. Hay que romper esa brecha entre la práctica y la teoría y así convertirse en modelo mediador para los procesos de aprendizaje

\subsection{Competencias del profesor universitario: Garantía de calidad en la docencia}

Ya definido de manera general el concepto de competencias, se mostrará algunas de las que deben caracterizar la función de los maestros en la educación superior. Estas según Esteban y Menjívar (2011), no se pueden centrar solamente en una función laboral, ni en la apropiación del conocimiento de acuerdo a su área disciplinar, sino que abarca un espectro más complejo que según el autor ocupa todo un conjunto de capacidades, que se desarrollan a través de procesos que conducen a la persona responsable a ser competente para realizar múltiples acciones como sociales, cognitivas, culturales, afectivas, laborales y productivas.

Uno de los autores más relevantes sobre el tema de competencias es Marchesi (2007), quien afirma que para ser un docente competente en su función en la universidad debe tener las siguientes características:

* Ser competente para favorecer el deseo de saber de los alumnos y para ampliar conocimientos

- Comprometer a los alumnos en sus aprendizajes

* Ser capaces de responder a la diversidad de los alumnos

* Estar preparado para incorporar la lectura en la actividad educadora

Ser capaz de incorporar las tecnologías de la información a la enseñanza; 
Vol. 1

No. 1

Jul - Dic 2016

ISSN: $2590-9215$ (En línea)

PP: $81-90$
* Estar preparado para velar por el desarrollo afectivo de los alumnos y por la convivencia

* El desarrollo emocional de los alumnos

* La convivencia en las aulas

* Ser capaz de favorecer la autonomía moral de los alumnos

* Educar en valores y para la ciudadanía

* Ser capaz de desarrollar una educación multicultural y encuentro entre las culturas

* Trabajar en colaboración y en equipo con los grupos de colegas (p. 42)

Otros autores argumentan que existen dos tipos de competencias que debe desarrollar el profesor universitario: las competencias individuales y las competencias colectivas.
Las primeras en la dimensión del saber actuar hacen relación a resolver situaciones en el ámbito pedagógico, dominio y las transferencias de los conocimientos, comunicación efectiva entre todos los actores educativos y a autogestión personal y profesional.

Lasegunda,esdecirlascolectivassemanifiestan cuando el docente consiguen acuerdos sobre un problema, meta a alcanzar, o situación a mejorar, se logra un mismo entendimiento sobre determinada información, se eligen los modos de cooperación apropiados para las diversas formas de organización y se aprende de las experiencias (Jabif, 2007).

A manera de cierre de esta categoría en el siguiente cuadro se pueden evidenciar algunas de las competencias y perfiles que debe tener un profesor universitario desde la perspectiva de diferentes autores que han realizado trabajos rigurosos en el área.

Tabla No. 3. Competencias y perfiles del docente universitario.

\begin{tabular}{|c|c|}
\hline Autor & Rasgos del perfil ideal \\
\hline $\begin{array}{l}\text { Barr y Tagg } \\
(1995)\end{array}$ & $\begin{array}{l}\text { - Sean diseñadores de ámbitos de aprendizaje. } \\
\text { - } \quad \text { Desarrollen la competencia y el talento de cada estudiante. } \\
\text { - Interactúen en equipos interdisciplinares e interdepartamentales. } \\
\text { - Promuevan la evaluación externa de los aprendizajes. }\end{array}$ \\
\hline $\begin{array}{l}\text { Carneiro } \\
2006\end{array}$ & $\begin{array}{l}\text { - Un saber avanzado en recursos curriculares y desarrollo de materiales } \\
\text { didácticos "propiedad" del profesor. } \\
\text { - Ser cada vez más "inventor" y "creador" de sus materiales de } \\
\text { enseñanza-aprendizaje. } \\
\text { Poseer una literatura tecnológica que sobrepase la mera "alfabetización } \\
\text { digital", a fin de entrar en los dominios de las competencias en el uso de } \\
\text { las nuevas TIC's. } \\
\text { Participar en redes de trabajo docente, presenciales y virtuales. }\end{array}$ \\
\hline $\begin{array}{l}\text { Trespalacios } \\
2008\end{array}$ & $\begin{array}{l}\text { - Trasciende la propuesta documental del curriculum formal y la pone en } \\
\text { relación con el entorno. } \\
\text { - Carga de significado la propuesta curricular al moverse en el "deber } \\
\text { ser", "deber saber" y "deber hacer". } \\
\text { Piensa, habla, siente y reflexiona sobre sí mismo, y se recrea en el } \\
\text { currículo, reconociendo su propia subjetividad, manteniéndose vigilante } \\
\text { sobre su actuar. } \\
\text { Influye de manera consciente desde un "darse cuenta" en el entorno } \\
\text { axiológico en que los individuos interaccionan pedagógicamente. } \\
\text { Potenciar la configuración de sujetos sociales capaces de intervenir la } \\
\text { realidad. }\end{array}$ \\
\hline $\begin{array}{l}\text { Zabalza } \\
(2003) y \\
\text { Camargo } \\
(2008)\end{array}$ & $\begin{array}{l}\text { - Conozcan las prácticas más adecuadas en los diferentes modelos de } \\
\text { aprendizaje. } \\
\text { - Conozcan las ventajas y el potencial de las nuevas TIC's. } \\
\text { - Slanifiquen el proceso E-A. } \\
\text { - Se comuniquen y relacionen con los alumnos. } \\
\text { - Reflexionen e investiguen sobre la enseñanza. } \\
\text { - Se identifiquen con la institución y trabajen en equipo. }\end{array}$ \\
\hline $\begin{array}{l}\text { Proyecto } \\
\text { Alfa-Tuning }\end{array}$ & $\begin{array}{l}\text { - Es un gran facilitador que pone en manos de los estudiantes recursos, } \\
\text { información, métodos y herramientas. }\end{array}$ \\
\hline
\end{tabular}

- Crea ambientes y les acompaña brindándoles asistencia a lo largo de todo el proceso.

- Eleva la motivación y compromiso de los estudiantes.

- Es un facilitador de procesos de desarrollo humano

- Es un asesor científico y metodológico. 


\section{Investigación y calidad de la educación}

Antes de iniciar con la categoría de calidad de la educación desde las perspectivas de la investigación, se abordará de manera general el concepto y el beneficio de la investigación en las dinámicas de la educación superior.

La investigación en su concepto general, se refiere a las actividades sistemáticas y racionales que un sujeto pone en marcha para llegar a un resultado, esto es, una nueva forma de comprender o explicar y de la misma forma controlar y predecir un hecho o un evento del mundo, y por tanto, darle significado a algo que no era visible, algo que en sentido estricto no existía para la humanidad. (Vanegas, Vélez, \& Vidarte, 2010). Este concepto en cierto sentido lo que pretende es mostrar el papel de la investigación como una manera de llenar los vacíos teóricos o epistemológicos de determinado problema.

La investigación en ciencias sociales concretamente se refiere según Bonilla, Hurtado y Jaramillo (2009) a la manera de cuantificar e interpretar la complejidad de los fenómenos y las limitaciones que conlleva el estudio con seres humanos en situaciones sociales.

Algunos autores presentan el concepto de investigación relacionado con lo metodológico y señalan que la investigación involucra el estudio de los métodos, los procedimientos y las técnicas que se deben utilizar para obtener conocimientos, para buscar una explicaciones y para una comprensión o explicación científica de los fenómenos, así como también para solucionar los problemas que demandan o plantea la sociedad. (Hernández, Maquilón, \& Sáez, 2008).

Otros autores más tradicionales señalan que la investigación es un procedimiento reflexivo, sistemático, controlado y crítico que tiene por finalidad descubrir o interpretar los hechos y fenómenos, relaciones y leyes de un determinado ámbito de la realidad
(Ballén, Pulido \& Zúñiga, 2007). Sin embargo, este concepto ya abordado por otros investigadores; incluye un elemento importante que mencionan los autores y es el de abordar la investigación como
"una metodología de transfor- mación y aprendizaje; como una corriente de pensamiento que atrae hoy en día tanto a profe- sionales de distintas áreas como a empresarios, administradores, planificadores, políticos, educa- dores y académicos, enmarcada en la concientización del indi- viduo sobre la realidad social y la obligación que tiene de trans- formarla mediante la aplicación de los conocimientos" (p.16).

El término aprendizaje y transformación que se menciona son categorías muy importantes pues no se está hablando directamente del objeto de estudio o del contexto, si no del efecto que puede hacer los procesos de investigación en los sujetos que investigan o los que están aprendiendo a investigar, es decir, más que construir conocimiento con un método riguroso y sistemático, se está abordando un proceso de aprendizaje y de transformación del ser humano.

La investigación junto con la docencia y la extensión hacen parte de los pilares fundamentales de la Universidad, no se puede concebir una institución de educación superior sin la generación de procesos de investigación que dinamicen la construcción
No. 1

Jul - Dic 2016

ISSN: 2590-9215 (En línea) PP: $81-90$ de conocimiento y el cambio social fruto de estos procesos.

En Colombia el sistema de aseguramiento de la calidad exige a las IES como política gubernamentales para la apertura de programas, renovación de registros calificados y acreditación institucional y de alta calidad contemplar la investigación como un elemento necesario en sus estructuras 
Vol. 1

No. 1

Jul - Dic 2016

ISSN: 2590-9215

(En línea)

PP: $81-90$ curriculares. En el decreto 1295 de abril de 2010 en el numeral 5.5, aparece este requerimiento informando a las instituciones de educación superior que promuevan la investigación en sus estudiantes y generen procesos de investigación atendiendo la dinámica de los grupos de investigación (COLCIENCIAS) y la producción científica de los docentes orientadores de los programas. Sin embargo, a propósito de estas exigencias, se puede apreciar que una limitante para que las instituciones de educación superior sean de naturaleza investigativa son los escasos recursos económicos con los que cuentan las instituciones para esta actividad.

Por su parte el CNA (2013) concibe la investigación como factor de calidad atendiendo a aspectos relacionados con la formación investigativa en los estudiantes en el nivel de pregrado, docentes dedicados a la investigación, producción científica de los profesores, tener reconocimiento de grupos de investigación por parte de COLCIENCIAS $\mathrm{u}$ otra institución y unas políticas por parte de la institución para la financiación de la investigación.

Desde otra mirada fuera de lo normativo, es innegable que la investigación es un determinante de la calidad de la educación superior y que además de generar conocimiento, "esta debe suplir las necesidades profesionales y ocupacionales de su entorno laboral con el cual mantiene estrecha relación" (Lago et al., p. 16).

Como se mencionó anteriormente la investigación está presente de acuerdo a la naturaleza de la misma institución, algunas instituciones de educación superior toman como objetivo principal la formación y no ven la investigación como un elemento potente de la transformación y de mejora en la calidad del servicio. En este sentido la investigación desde el punto de vista de la calidad tiene repercusiones importantes en diversos aspectos, por ejemplo, la investigación como eje fundamental en la transformación de las prácticas pedagógicas del docente, pues el ejercicio investigativo concede al profesor mayor profundidad de conocimientos, permite apreciar el papel que desempeña la investigación en el desarrollo de su vida y lo ayuda a mejorar sus métodos ${ }^{1} \mathrm{y}$ resolver problemas educacionales. La investigación le abre un nuevo e interesante mundo en

el cual puede experimentar un continuo mejoramiento personal y profesional y esto es una garantía de calidad para la institución. Sin embargo, "el hecho de que un profesor universitario se destaque en la actividad investigadora no implica necesariamente que posea las habilidades para una buena docencia" (Parra, 2004, p. 61).

No obstante, el hecho de que el profesor no haga investigación tampoco lo hace un mal docente, solo que le provee múltiples herramientas para la función educadora $y$ optimiza el aprendizaje de los estudiantes a su cargo.

\section{Gestión y calidad de la educación superior}

La gestión es otro elemento fundamental en la dinámica interna y externa de las instituciones de educación superior. La gestión a modo general está definida como un conjunto de operaciones que se realizan para dirigir y administrar un negocio o una empresa: una buena gestión hace que las empresas funcionen.

En el ámbito educativo la gestión según Lavín y del Solar (2000), corresponde a toda actividad relacionada con los elementos administrativos y financiaros de una institución, así como a la forma en la cual

\footnotetext{
${ }^{1}$ En cuanto a una mejora en la enseñanza de los saberes o volver enseñable un saber desde la práctica del docente es importante citar a Parra, $(2004$, p. 62) quien señala que “...la investigación didáctica es connatural al quehacer universitario, la cual se entiende aquí como aquella cuyo objeto son los procesos de formación, específicamente de enseñanza aprendizaje, y los demás procesos curriculares propios de la función docente"
} 
los directivos, docentes y los estudiantes se organizan. Del mismo modo se refiere a la fijación de calendarios, fijación de horarios, distribución de espacios y otros elementos organizativos. De igual manera, se configura como el conjunto de acciones, relacionadas entre sí que emprende el equipo directivo de una institución para promover y posibilitar la consecución de la intencionalidad en - con la comunidad educativa.

Lavín y Del solar (200), plantean que la gestión educativa o gestión escolar posee básicamente 6 dimensiones que se mencionan a continuación:

Dimensión pedagógica curricular: Son los fines específicos o razón de ser de la institución en la sociedad.

O Dimensión administrativa - financiera: Es la distribución del tiempo, el espacio, el recurso humano y los aspectos financieros de la institución

Dimensión organizativa - operacional: Se refiere a la organización de los subsistemas de la institución, es decir organización del cuerpo de docente y directivos, departamentos administrativos, departamentos académicos, comisiones, entre otros.

Dimensión comunitaria: Aborda la relación de la institución con el sector externo (sociedad, empresa, organizaciones culturales)

Dimensión de convivencia: Es lo que garantiza el clima organizaciones entre los diferentes actores educativos. (Docentes, administrativos, estudiantes, directivos). Establece las normas internas del actuar en la institución

Dimensión sistémica: Es la relación que establece la institución con otras instituciones del sistema educativo (otras universidades, secretarias de educación, etc.). (p.59)

Uno de los autores que más se ha referido a la gestión como factor de calidad en la educación superior es Municio, quien plantea un enfoque fundamental al respecto: la gestión de la educación por procesos; este concepto se incrusta en la gestión de la calidad educativa como un nuevo modelo para

"hacer frente a la demandas de la sociedad actual. Por una parte se aporta tecnología para lograr organizaciones más eficientes $y$, por otra, la orientación a las personas implicadas como razón esencial de la organización y de los procesos. La confluencia se traduce por procesos de mejora continua y el resultado en el valor agregado por la acción del proceso" (Municio, 2000, p. 113).

En la siguiente figura se puede observar a manera general el esquema de la gestión por procesos:

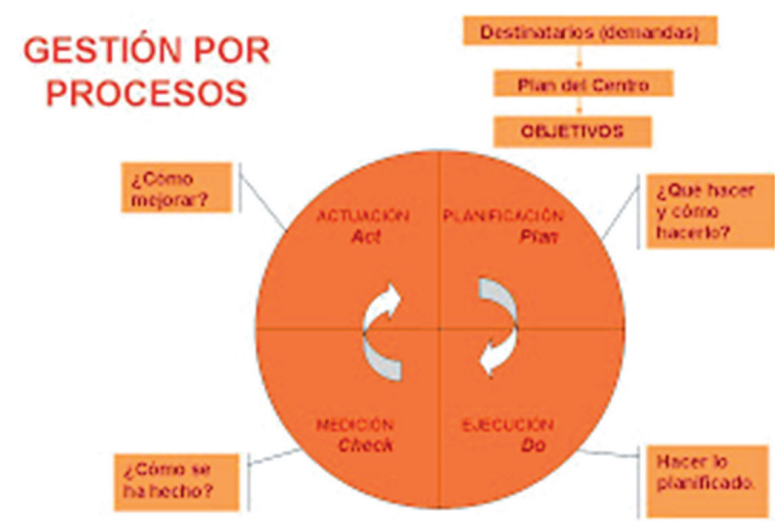

Figura 1. Gestión por procesos. Fuente: Beltrán, Carmona, Carrasco, Rivas y Tejedor (2002)

\section{Conclusiones}

Gran parte de los discursos sobre la docencia suponen que esta tiene su fundamento en el conocimiento especializado de las disciplinas y las profesiones. Sus propósitos consisten en formar profesionales, promover el ejercicio de la ciudadanía y el desarrollo integral de las personas que desempeñan el rol de 
Vol. 1

No. 1

Jul - Dic 2016

ISSN: 2590-9215

(En línea)

PP: $81-90$ estudiantes. Así mismo, se puede afirmar que la docencia es una actividad cotidiana que va configurando un sistema de toma de decisiones, actuación y valoraciones que se convierten en rasgos distintivos e imaginarios fuertemente instituidos en las instituciones de educación superior. En esa línea, los modos de actuar frente al conocimiento, a los estudiantes y al contexto social, se pueden estudiar bajo la denominación de docencia cuyos propósitos educativos consisten en la contextualización de las características de la ciencia contemporánea y las tendencias de la época actual, coherentes con la formación integral y proyectada a largo plazo.

La investigación por su parte, comprende el desarrollo de una actitud, el dominio de un método y el ejercicio de una práctica. El desarrollo de una actitud investigativa es posible de lograr a través de las experiencias curriculares con la ejercitación intensa de la capacidad racional y lógica de la mente del estudiante en el momento de abordar los hechos científicos y sociales. De manera cómo lo afirma (De Zubiría, 2006), se desarrollan competencias de análisis y crítica y la capacidad de formular preguntas alrededor de los fenómenos en estudio antes que la recepción pasiva de las respuestas sobre los mismos, característica de la enseñanza tradicional.

Por otra parte, la gestión institucional es un factor determinante de calidad en la universidad. Todos los procesos y objetivos misionales descansan en la conducción adecuada de los organismos administrativos. Es por esto que el futuro de las universidades, su sobrevivencia y posicionamiento en un medio competitivo y abierto como el que ya se perfila, dependerá cada vez más de la capacidad que tengan las instituciones para transformarse y operar por sí misma bajo una conducción eficaz.

\section{Referencias bibliográficas}

Arrufat, M., Sánchez, V. \& Santiuste, E. (2010). El futuro docente ante las competencias en el uso de las tecnologías de la información y comunicación para enseñar. Tecnología educativa, 34 (1), 7 - 19.

Beneitone et al. (2007). Reglexiones y perspectivas de la Educación Superior en America Latina. Informe Final Proyecto Tuning America Latina. Bilbao: Universidad de Deusto.

Beltrán, J., Carmona, M., Carrasco, R., Rivas, M., \& Tejedor, F. (2002). Guía para una gestión basada en procesos. Instituto Andaluz de Tecnología. Govern de les Illes Balears.

Ballén, M., Pulido, R. \& Zúñiga, L. (2007). Abordaje hermenéutico de la investigación cualitativa: Teorías, Proceso, Temáticas. Bogotá: Universidad Cooperativa de Colombia.

Bonilla, E., Hurtado, J. \& Jaramillo, C. (2009). La investigación. Aproximación a la Construcción del conocimiento cientifico. México: Alfaomega.

Bozu, Z., \& Herrera, P. (2009). El profesorado universitario en la sociedad del conocimiento: competencias profesionales docente. Formación e Innovación Educativa Universitaria, 2(2), 221-231.

CNA. (2013). Acreditación de programas de pregrado. Colombia

De Vincenzi, A. (2009). Concepciones de enseñanza y su relación con las prácticas docentes: un estudio con profesores universitarios. Educación y educadores, 12(2), 89 - 101. 
De Zubiria, S. (2006). Modelos pedagógicos. Hacia una pedagogía dialogante. Bogotá: Magisterio.

Esteban, R. \& Menjívar, S. (2011). Una mirada internacional a las competencias docentes Universitarias. Barcelona: Octaedro.

García, E., Martín, S., Amblàs, G., Puchades, F., Sallarés, J., \& Bugeda, G. (2010). La evaluación de la actividad docente del profesorado en el marco del EEES. Aula Abierta, 38(2), 29-40.

Guzmán, I., \& Marín, R. (2011). La competencia y las competencias docentes: reflexiones sobre el concepto y la evaluación. Revista electrónica interuniversitaria de formación del profesorado, 36, (14-1), 151-163.

Hernández, F., Maquilón, J., \& Sáez, J. (2008). El proceso de investigación y el análisis de datos en ciencias sociales. Murcia: Diego Marín.

Hernandez, A. (2009). Desarrollo y evaluación de competencias en educación superor. Madrid: Narcea.

Jabif, L. (2011). La Docencia Universitaria Bajo un Enfoque de Competencias. Chile: Universidad Austral.

Lago, D., López, E., Municio, P., Ospina, R., \& Vergara, G. (2013). La Calidad de la Educación Superior. ¿Un reto o una utopía?. Bogotá: Ecoe ediciones

Laudadío, J. (2012). Evaluación de estilos de enseñanza en la universidad: estudio preliminar de las propiedades psicométricas del cuestionario sobre la orientación docente del profesor universitario (CODPU). Psicología y ciencias Afines, 29(1), 79-93.
Lavín, S. \& Del Solar, S. (2000). El proyecto educativo institucional como herramienta de transformación de la vida escolar. Chile: LOM

López, N. (2001). La deconstrucción curricular. Colombia: Magisterio

Marchesi, Á. (2007). Sobre el bienestar de los docentes: Competencias, emociones $y$ valores. Madrid: Alianza. Manzi, J. (2011). La evaluación docente en Chile. Chile: MIDE UC.

Montenegro, I. (2005). Aprendizaje y desarrollo de competencias. Bogotá: Magisterio.

Municio, P. (2000). La evaluación de la calidad en educación. Madrid: Narcea-Consudec.

Osorio, J. (2011). Competencias docentes en la profesión académica en México. En: Jaik, A. \& Barraza, A. (Coord.). Competencias y educación: miradas múltiples de una relación. (pp. $146-$ 162). México: Redie.

Parra, C. (2004). Apuntes sobre la investigación formativa. Educación y educadores, 7 (77), 57 - 77.

Tobón, C. \& Tejada, S. (2006). El diseño del plan docente en Información y Documentación acorde con el Espacio Europeo de Educación Superior: un enfoque por competencias. Madrid: Universidad Complutense.

Tobón, C. (2008). La formación Basada en Competencias en la Educación Sueperior: Un enfoque complejo. Bogotá: Insituto CIFE.

Tejedor, F. J., \& García, A. (2010). Evaluación del desempeño docente. Revista española de pedagogía, 68(427), 439459. 
Vol. 1

No. 1

Jul - Dic 2016

ISSN: 2590-9215 (En línea)

PP: $81-90$
Tirado, M. (2009). Docencia universitaria y competencias didácticas. Perfiles educativos, 31(125), 76-87.

Vanegas, J., Vélez, C. \& Vidarte, J. (2010). Aproximación a los fundamentos y métodos de investigación. Manizales: UAM.

Villardón, L. (2006). Planificar desde competencias para promover el aprendizaje. Bilbao: Universidad de Deusto.

Zabalza, M. \& Zabalza, M. (2010). Planificación de la docencia en la universidad: Elaboración de las guías docentes de las materias. Madrid: Narcea. 